\title{
Association of rs780094 polymorphism of glucokinase regulatory protein with non-alcoholic fatty liver disease
}

\section{Saba Mohammadi}

Tabriz University of Medical Sciences

Safar Farajnia ( $\square$ farajnias@tbzmed.ac.ir)

Institute for Bioscience and Biotechnology Research Shady Grove https://orcid.org/0000-0002-60879147

\section{Fatemeh Mohseni}

Tabriz University of Medical Sciences

\section{Roghayyeh Baghban}

Tabriz University of Medical Sciences

Masoud Shadmand

Tabriz University of Medical Sciences

\section{Research note}

Keywords: NAFLD, rs780094 polymorphism, biochemical parameters, demographic parameters

Posted Date: August 31st, 2019

DOI: https://doi.org/10.21203/rs.2.13792/v1

License: (c) (i) This work is licensed under a Creative Commons Attribution 4.0 International License. Read Full License

Version of Record: A version of this preprint was published at BMC Research Notes on January 10th, 2020. See the published version at https://doi.org/10.1186/s13104-020-4891-y. 


\section{Abstract}

Objective GCK rs780094 polymorphism is a single nucleotide polymorphism that has been associated with obesity, type II diabetes and dyslipidemia in some populations, conditions that highly related to NAFL etiology. The present study aimed to evaluate the relationship between NAFLD and rs780094 polymorphism in patients with NAFLD in Tabriz city, northwest of Iran. The rs780094 polymorphism was determined in 74 patients with NAFLD by PCR-RFLP technique. Demographic information was collected using a questionnaire and biochemical analysis was performed using standard laboratory methods.

Results There were a significant difference between case and control subjects for alanine aminotransferase, aspartate aminotransferase, HDL-C and triglycerides $(P<0.05)$. Analysis by PCR-RFLP method revealed that there were no significant differences between NAFLD and healthy subjects for rs780094 polymorphism in the study population. The results of this study indicated that rs780094 polymorphism is not associated with NAFLD in subjects from Tabriz city.

\section{Background}

Non-alcoholic fatty liver disease (NAFLD), was first identified in 1980 by Ludwig et al, through fatty infiltration of the liver among non-alcoholic patients [1]. NAFLD is considered as a major public health concern due to increasing prevalence of obesity in both children and adults. The disease is diagnosed by hepatic steatosis or presence of fat in liver parenchyma without inflammation and also in the absence of excessive alcohol consumption. NAFLD is also known as a hepatic component of metabolic syndrome [2] which includes a broad range of clinic pathological conditions ranging from simple steatosis to non-alcoholic steatohepatitis (NASH) [3]. The progressed NASH is identified through cellular necrosis and inflammatory infiltration that can subsequently create complications such as cirrhosis, liver failure and hepatocellular carcinoma [4].

Genetic variants, as likely etiological factors, are investigated for NAFLD due to its familial clustering [5-8]. Some research studies have shown a strong correlation between usual missense variant (rs738409) in the patatin-like phospholipase domain-containing protein 3 (PNPLA3) gene and susceptibility to NAFLD and NASH [9].

Glucose-phosphorylating enzyme glucokinase, is responsible for hepatic glucose metabolism and activation of hepatic lipogenesis. The expression of Glucokinase is regulated by GCKR [10]. It has shown that GCKR, encoded by GCKR gene, allosterically binds to GCK and regulates its activity. Genome-wide association studies (GWAS) have 
recognized a single-nucleotide polymorphisms (SNPs) which increases the risk of NAFLD. It has shown that rs780094 polymorphism of GCKR is related to triglyceride levels [8, 11, 12]. Also, an association between rs780094 polymorphism of GCKR and diabetes, particularly in Asian population has reported in a previous study [13]. Moreover, population studies showed that NAFLD is severely related to obesity, insulin resistance/type

II diabetes mellitus and dyslipidemia [14-17]. Thus, we hypothesized the presence of a possible relationship between NAFLD and rs780094 polymorphism. The presence of strong association between NAFLD, with diabetes mellitus and hepatic triglyceride accumulation and also association between rs780094 polymorphism with diabetes and triglyceride levels supported our hypothesis. The present study investigated the relationship between NAFLD and rs780094 polymorphism in patients with NAFLD in subjects from Tabriz city in northwest of Iran.

\section{Methods}

\section{Subjects and samples}

This case-control study was conducted on 74 patients with NAFLD that were confirmed by both sonography and high levels of liver enzymes. Also, 72 subjects without NAFLD were considered as control. The patients were randomly selected from patients in Gastrointestinal clinics. The subjects were informed about the purpose of the study and a written informed consent was obtained. The inclusion criteria were similar in the case and control subjects, as follows; mean age 20-50 years and mean body mass index (BMI) 25$39.9 \mathrm{~kg} / \mathrm{m}^{2}$. The exclusion criteria were also as follows; age ranging $>50$ years and $<20$ years, BMI $<25 \mathrm{~kg} / \mathrm{m}^{2}$, having hemochromatosis, Wilson and autoimmune diseases, tobacco and alcohol consumption, the use of hepatotoxic and psychotic drugs, weight loss diets over last 3 months, gland disorders, pregnancy and lactation, intestinal bypass surgery, acute and chronic liver diseases and viral hepatitis. 


\section{Samples preparation and Biochemical analysis}

Blood samples were collected from case and control subjects and transferred to molecular biology laboratory of biotechnology research center. The biochemical parameters consisting of alanine aminotransferase (ALT), aspartate aminotransferase (AST), low density lipoprotein (LDL) cholesterol, high density lipoprotein (HDL) cholesterol, total cholesterol, and plasma triglyceride level were analyzed using standard laboratory techniques.

\section{Collection of anthropometric data}

The information related to age, sex, drug consumption, cigarette smoking, etc. were collected by questionnaire. BMI was defined as weight $/$ height ${ }^{2}\left(\mathrm{~kg} / \mathrm{m}^{2}\right)$. Waist and hip circumference and waist-to-hip ratio were also measured.

\section{DNA extraction and Genotyping}

DNA was extracted from blood samples using salting-out method. Briefly, $500 \mu \mathrm{l}$ of bloodsamples were transferred to the $15 \mathrm{ml}$ tubes and the red blood cells were lysed by washing three times with lysis buffer. Then, $500 \mathrm{\mu l}$ of DNG-Plus reagents (Cinnagen co, Iran) was added to the cell pellet and slowly stirred to obtain the homogenized sample. DNA was then precipitated by $350 \mu$ of isopropanol, washed with ethanol $75 \%$ and dissolved in $50 \mu \mathrm{l}$ distilled water. The extracted DNA was analyzed using electrophoresis on $1 \%$ agarose gel and spectrophotometry.

RFLP-PCR was used to determinate the genotypes of subjects for rs738409 polymorphism. The DNA extracted from subjects was amplified with the following primers, Forward: 5'AGCTCACGCTGGAACTTCTG -3' and Reverse: 5'- CATGTTGGCTAGGCTTGTTGAG -3', using Taq DNA polymerase. PCR reaction was performed in a final volume of $25 \mu \mathrm{l}$ including; $10 \mathrm{pm}$ of each primer, $100 \mathrm{ng}$ of genomic DNA, 1U of Taq DNA polymerase and $22 \mu \mathrm{l}$ of PCR master mix. The PCR program for DNA amplification was as follows; initial denaturation at $94^{\circ} \mathrm{C}$ for 4 minutes, 35 cycles of denaturation at $94^{\circ} \mathrm{C}$ for 1 minute, annealing at $53^{\circ} \mathrm{C}$ for 1 minute, extension at $72^{\circ} \mathrm{C}$ for 45 s and final extension at $72^{\circ} \mathrm{C}$ for 5 
minutes. After PCR, $10 \mu \mathrm{l}$ of PCR products were digested with $1 \mathrm{U}$ of PscI enzyme for 1 hour at $37^{\circ} \mathrm{C}$ and visualized on $2 \%$ agarose gel, after staining with safestain. The 100-bp DNA ladder was used for determining the size of digested product. After electrophoresis, genotypes GG at 311 bp, CC at 194bp and 117bp and CG at 311 and 194bp were detected.

\section{Statistical analysis}

Statistical analysis was performed using SPSS software Version 23. Qualitative and quantitative data were expressed as Mean ( \pm standard deviation) and frequency (\%), respectively. The Mann-Whitney $U$ test and Independent t-test were used for analysis of the data. $P<0.05$ was considered as significant.

\section{Results}

\section{Demographic and clinical characteristics}

Demographic and clinical data of the subjects have been demonstrated in Table 1. There were significant differences between control and case group for HDL-C, triglycerides, ALT and AST $(P<0.05)$, so that patients with NAFLD showed higher concentrations of triglycerides, ALT and AST and lower concentration of HDL-C compared to control group $(P<0.05)$.

Table 1 Demographic and clinical data of the subjects 


\begin{tabular}{lccc}
\hline characteristics & Control (N=72) & Case (N=74) & $\boldsymbol{P}$ \\
\hline Sex (men \%) & 36.1 & 48.6 & $\mathrm{~ns}$ \\
Sex (women \%) & 63.9 & 51.4 & $\mathrm{~ns}$ \\
Age (years) & $38.88 \pm 8.25$ & $40.54 \pm 8.42$ & $\mathrm{~ns}$ \\
BMI (kg/m ${ }^{2}$ ) & $31.51 \pm 4.09$ & $31.90 \pm 4.18$ & $\mathrm{~ns}$ \\
Waist circumference (cm) & $100.2 \pm 8.75$ & $103.36 \pm 9.31$ & $\mathrm{~ns}$ \\
Hip circumference (cm) & $111.86 \pm 8.05$ & $112.16 \pm 8.13$ & $\mathrm{~ns}$ \\
Waist-to-hip ratio & $0.897 \pm 0.065$ & $0.922 \pm 0.052$ & $\mathrm{~ns}$ \\
Weight (kg) & $84.66 \pm 12.15$ & $86.44 \pm 11.14$ & $\mathrm{~ns}$ \\
Height (cm) & $164.04 \pm 10.19$ & $164.96 \pm 10.49$ & $\mathrm{~ns}$ \\
Cholesterol (mg/dl) & $187.40 \pm 29.33$ & $183.08 \pm 37.03$ & $\mathrm{~ns}$ \\
Triglycerides (mg/dl) & $140.71 \pm 75.84$ & $173.55 \pm 76.33$ & $*$ \\
HDL-C (mg/dl) & $48.08 \pm 11.78$ & $43.09 \pm 11.43$ & $*$ \\
LDL-C (mg/dl) & $110.98 \pm 26.64$ & $105.03 \pm 34.75$ & $\mathrm{~ns}$ \\
ALT (IU/L) & $26.29 \pm 9.41$ & $49.99 \pm 26.16$ & $*$ \\
AST (IU/L) & $22.97 \pm 6.11$ & $32.99 \pm 14.97$ & $*$ \\
Fasting glucose (mg/dl) & $90.17 \pm 10.16$ & $90.47 \pm 11.27$ & ns \\
\hline & & &
\end{tabular}

*: $P<0.05$; ns: non-significant or $\mathrm{P}>0.05$

Genotypic and allelic frequency of rs780094 polymorphism of GCKR

The distribution of the GCKR rs780094 genotype for the case and control groups has shown in table 2. Analysis of the results indicated that there was no significant difference between case and control groups for CT and TT genotypes. Hovever, compared to the normal healthy control subjects, the frequency of CC genotypes was significantly lower in NAFLD patients (Table 2).

Table 2 Genotypic and allelic frequency (\%) of GCKR rs780094 


\begin{tabular}{lccc}
\hline & $\begin{array}{c}\text { Control } \\
\text { N=72 }\end{array}$ & $\begin{array}{c}\text { Case } \\
\text { N=74 }\end{array}$ & $P$ \\
\hline CC genotype & 31.9 & 18.9 & $\mathrm{~ns}$ \\
CT genotype & 47.2 & 48.6 & $\mathrm{~ns}$ \\
TT genotype & 20.8 & 32.4 & $\mathrm{~ns}$ \\
T allele & 0.44 & 0.57 & $\mathrm{~ns}$ \\
C allele & 0.56 & 0.43 & $\mathrm{~ns}$ \\
\hline
\end{tabular}

*: $P<0.05$; ns: non-significant or $\mathrm{P}>0.05$

Relation of demographic and clinical characteristics with GCKR rs780094

Table 3 shows a correlation between demographic and clinical characteristics of subjects and GCKR rs780094 genotype. Logistic regression showed that there was not any correlation between demographic and clinical characteristics and GCKR rs780094 genotype; indicating lack of significant role of GCKR rs780094 genotype in NAFLD in the study population.

Table 3 Relation of demographic and clinical characteristics with GCKR rs780094 genotype in patients with NAFLD $(\mathrm{N}=74)$ 


\begin{tabular}{lccccc}
\hline characteristics & CC & CT & TT & $\boldsymbol{\beta}$ & $P$ \\
& & & & Coefficient & \\
\hline Sex (men \%) & 64.3 & 38.9 & 50 & - & $\mathrm{ns}$ \\
Sex (women \%) & 35.7 & 61.1 & 50 & - & $\mathrm{ns}$ \\
Age (years) & $40.43 \pm 8.67$ & $42.58 \pm 8.10$ & $38.29 \pm 8.53$ & -0.027 & $\mathrm{~ns}$ \\
BMI (kg/m ${ }^{2}$ ) & $30.70 \pm 3.05$ & $31.30 \pm 4.19$ & $32.16 \pm 4.59$ & 0.057 & $\mathrm{~ns}$ \\
Waist circumference & $100.0 \pm 7.33$ & $106.0 \pm 10.91$ & $101.0 \pm 7.53$ & -0.033 & $\mathrm{~ns}$ \\
(cm) & & & & & \\
Hip circumference (cm) & $108.0 \pm 7.14$ & $115.0 \pm 8.36$ & $111.0 \pm 7.10$ & 0.011 & $\mathrm{~ns}$ \\
Weight (kg) & $82.44 \pm 9.56$ & $88.78 \pm 11.59$ & $88.78 \pm 11.59$ & 0.136 & $\mathrm{~ns}$ \\
Height (cm) & $167.43 \pm 8.93$ & $163.28 \pm 10.81$ & $165.0 \pm 10.50$ & 0.103 & $\mathrm{~ns}$ \\
Cholesterol (mg/dl) & $176.64 \pm 45.27$ & $185.14 \pm 35.06$ & $182.17 \pm 33.75$ & -0.037 & $\mathrm{~ns}$ \\
Triglycerides (mg/dl) & $184.71 \pm 68.55$ & $172.64 \pm 88.37$ & $187.08 \pm 39.37$ & 0.007 & $\mathrm{~ns}$ \\
HDL-C (mg/dl) & $43.00 \pm 10.94$ & $43.97 \pm 11.79$ & $43.08 \pm 11.07$ & -0.007 & $\mathrm{~ns}$ \\
LDL-C (mg/dl) & $96.70 \pm 40.81$ & $107.77 \pm 29.91$ & $101.67 \pm 35.29$ & 0.051 & $\mathrm{~ns}$ \\
ALT (IU/L) & $52.21 \pm 20.25$ & $44.11 \pm 29.10$ & $56.75 \pm 23.80$ & 0.012 & $\mathrm{~ns}$ \\
AST (IU/L) & $36.29 \pm 10.97$ & $29.61 \pm 12.41$ & $36.67 \pm 19.04$ & -0.010 & $\mathrm{~ns}$ \\
Fasting glucose (mg/dl) & $90.57 \pm 8.57$ & $92.06 \pm 10.08$ & $88.00 \pm 13.32$ & -0.005 & $\mathrm{~ns}$ \\
\hline & & & & &
\end{tabular}

\section{ns: non-significant or $\mathrm{P}>0.05$}

\section{Discussion}

Genome-wide association studies have shown that polymorphisms in the glucokinase regulatory protein are related to triglyceride level, obesity, insulin resistance/type II diabetes mellitus and dyslipidemia [14-17], common conditions associated with NAFLD disorder. Therefore, we aimed to investigate the relationship between the GCKR rs780094 variant and NAFLD in patients in the city of Tabriz, northwest of Iran.

The results of present study indicated that TT genotype had higher frequency in NAFLD cases compared to the control group, although this difference was not significant $(P>0.05)$. 
This finding was against report from china. Yang et al. in 2011. [11] reported that the GCKR variants could be considered as a risk factor for NAFLD.

A meta-analysis has also shown that GCKR rs780094 $\mathrm{T}$ allele is a protective factor against diabetes and obesity [11]. Some other studies has shown a significant association between GCKR rs780094 and NAFLD [18]. However, the lack of relationship between the GCKR rs780094 genotype and the NAFLD, in the present study, might be attributed to the different ethnic background of the studied groups. Also in previous studies, liver biopsy has been used as sample, while in this study the PBMC was used for DNA isolation.

In the current study, there was no any association between GCKR rs780094 and anthropometric characteristics of the patients. It has known that anthropometric parameters not only related to the genetic background, but also dependent to lifestyle. In a study on Iranian population, it has been shown a relationship between NAFLD and age, sex, obesity, metabolic syndrome, triglycerides, higher glucose, blood pressure and waist circumference [19]. Bhatt et al.[20] reported lack of any significant correlation between age and NAFLD in Asian Indians. Consistent with our findings, Lin et al. [21] A study on Taiwanese children reported lack of significant association between NAFLD, age, BMI and waist circumference.

ALT and AST are known as liver biomarkers and liver injuries in NAFLD can disturb ALT and AST levels. On the other hand, liver is known as a metabolizing organ for lipids, thus NAFLD, as a liver disorder, can disturb lipid levels. Some studies reported conflicting results for patients with NAFLD. Our results indicated significant changes in HDL-C, triglycerides, AST and ALT as risk factors. Consistent with our results, Bhatt et al. [20] and Hotta et al. [22] reported that ALT and AST were significantly higher in NAFLD group compared to healthy controls in Asian populations.

In contrast to our findings, Petta S et al. [23] reported that HDL, triglycerides, cholesterol and ALT did not show significant difference between case and control groups. In another investigation, Shen et al.[24] could not find significant difference for fasting glucose, cholesterol and triglycerides. 


\section{Conclusions}

The results of our study indicated that GCKR rs780094 TT genotype is not responsible for NAFLD in the studied population. Our results also showed that age, sex, BMI, cholesterol, fasting glucose, LDL-C, waist circumference, hip circumference and Waist-to-hip ratio were not significantly different in case and control groups. Changes in triglycerides, ALT, AST, and HDL-C were found as risk factors of NAFLD in the study groups.

\section{Limitations}

This study was a cross-sectional study, so a large sample size was required to consolidate findings of this study.

\section{Declarations}

\section{Availability of data and materials}

The dataset analyzed during the current study is available from the corresponding author on reasonable request.

\section{Abbreviations}

PCR: Polymerase chain reaction

RFLP: Fragment length polymorphism

NAFLD: Non-alcoholic fatty liver disease

PNPLA3: Phospholipase domain-containing protein 3

NASH: Non-alcoholic steatohepatitis

GCKR: Glucokinase regulatory

SNPs: Single-nucleotide polymorphisms

GWAS: Genome-wide association studies

ALT: Alanine aminotransferase

AST: Aspartate aminotransferase

LDL: Low density lipoprotein

HDL: high density lipoprotein 


\section{Acknowledgements}

This study was supported by Drug Applied Research Center, Tabriz University of Medical

Science. We thank our colleagues in Drug Applied Research Center who provided insight and expertise that greatly assisted the research.

\section{Funding}

This study was supported by a grant from Drug Applied Research Center, Tabriz University of Medical Science, Tabriz, Iran.

\section{Author information}

\section{Affiliations}

${ }^{1}$ Drug Applied Research Center, Tabriz University of Medical Sciences, Tabriz, Iran

${ }^{2}$ Biotechnology Research Center, Tabriz University of Medical Sciences, Tabriz, Iran Drug

${ }^{3}$ Nutrition Research Center, Department of Community Nutrition, School of Nutrition, Tabriz University of Medical Sciences, Tabriz, Iran

\section{Contributions}

SF conceived and designed the study. SM collected and analyzed the data and drafted the manuscript. FM, RB and MSH involved in the fieldwork, data collection and interpretation. All the authors reviewed the manuscript and approved the final draft.

\section{Corresponding author}

Correspondence to Safar Farajnia

\section{Ethics declarations}

\section{Ethics approval and consent to participate}

Ethical clearance was obtained from the Drug Applied Research Center, Tabriz University of medical science. Prior to data collection, written informed consent was obtained from 
the patients.

\section{Consent for publication}

Not applicable

\section{Competing interests}

The authors declare that they have no any competing interests.

\section{References}

1. Ludwig J, Viggiano TR, Mcgill DB, Oh B: Nonalcoholic steatohepatitis: Mayo Clinic experiences with a hitherto unnamed disease. In: Mayo Clinic Proceedings: 1980; 1980: 434-438.

2. Chalasani N, Younossi Z, Lavine JE, Diehl AM, Brunt EM, Cusi K, Charlton M, Sanyal AJ. The diagnosis and management of non-alcoholic fatty liver disease: Practice Guideline by the American Association for the Study of Liver Diseases, American College of Gastroenterology, and the American Gastroenterological Association. Hepatology 2012, 55(6):2005-2023.

3. Andy SY, Keeffe EB. Nonalcoholic fatty liver disease. Gastroenterol disord 2002, 2(1):B11-19.

4. Ioannou GN, Haigh WG, Thorning D, Savard C. Hepatic cholesterol crystals and crownlike structures distinguish NASH from simple steatosis. J Lipid Res 2013, 54(5):13261334.

5. Schwimmer JB, Celedon MA, Lavine JE, Salem R, Campbell N, Schork NJ, Shiehmorteza M, Yokoo T, Chavez A, Middleton MS. Heritability of nonalcoholic fatty liver disease. Gastroenterology 2009, 136(5):1585-1592.

6. Romeo S, Kozlitina J, Xing C, Pertsemlidis A, Cox D, Pennacchio LA, Boerwinkle E, Cohen JC, Hobbs HH. Genetic variation in PNPLA3 confers susceptibility to nonalcoholic fatty liver disease. Nature Genet 2008, 40(12):1461. 
7. Chalasani N, Guo X, Loomba R, Goodarzi MO, Haritunians T, Kwon S, Cui J, Taylor KD, Wilson L, Cummings OW. Genome-wide association study identifies variants associated with histologic features of nonalcoholic Fatty liver disease. Gastroenterology 2010, 139(5):1567-1576. e1566.

8. Speliotes EK, Yerges-Armstrong LM, Wu J, Hernaez R, Kim LJ, Palmer CD, Gudnason V, Eiriksdottir G, Garcia ME, Launer LJ. Genome-wide association analysis identifies variants associated with nonalcoholic fatty liver disease that have distinct effects on metabolic traits. PLoS Genet 2011, 7(3):e1001324.

9. Zain SM, Mohamed R, Mahadeva S, Cheah PL, Rampal S, Basu RC, Mohamed Z. A multi-ethnic study of a PNPLA3 gene variant and its association with disease severity in non-alcoholic fatty liver disease. Hum Genet 2012, 131(7):1145-1152.

10. Santoro N, Zhang CK, Zhao H, Pakstis AJ, Kim G, Kursawe R, Dykas DJ, Bale AE, Giannini C, Pierpont B. Variant in the glucokinase regulatory protein (GCKR) gene is associated with fatty liver in obese children and adolescents. Hepatology 2012, 55(3):781-789.

11. Yang Z, Wen J, Tao X, Lu B, Du Y, Wang M, Wang X, Zhang W, Gong W, Ling C. Genetic variation in the GCKR gene is associated with non-alcoholic fatty liver disease in Chinese people. Mol Biol Rep 2011, 38(2):1145-1150.

12. Bi M, Kao WHL, Boerwinkle E, Hoogeveen RC, Rasmussen-Torvik LJ, Astor BC, North KE, Coresh J, Köttgen A. Association of rs780094 in GCKR with metabolic traits and incident diabetes and cardiovascular disease: the ARIC Study. PloS one 2010, 5(7):e11690.

13. Fu D, Cong X, Ma Y, Cai H, Cai M, Li D, Lv M, Yuan X, Huang Y, Lv Z. Genetic polymorphism of glucokinase on the risk of type 2 diabetes and impaired glucose regulation: evidence based on 298, 468 subjects. PloS one 2013, 8(2):e55727.

14. Sanyal AJ. AGA technical review on nonalcoholic fatty liver disease. Gastroenterology 2002, 123(5):1705-1725.

15. Marchesini G, Brizi M, Morselli-Labate AM, Bianchi G, Bugianesi E, McCullough AJ, Forlani G, Melchionda N. Association of nonalcoholic fatty liver disease with insulin 
resistance. Am J Med 1999, 107(5):450-455.

16. Powell EE, Cooksley WGE, Hanson R, Searle J, Halliday JW, Powell W. The natural history of nonalcoholic steatohepatitis: a follow-up study of forty-two patients for up to 21 years. Hepatology 1990, 11(1):74-80.

17. Kawano Y, Cohen DE. Mechanisms of hepatic triglyceride accumulation in nonalcoholic fatty liver disease. J Gastroenterol 2013, 48(4):434-441.

18. Zain SM, Mohamed Z, Mohamed R. A common variant in the glucokinase regulatory gene rs780094 and risk of nonalcoholic fatty liver disease: A meta-analysis. $J$ Gastroenterol Hepatol 2015, 30(1):21-27.

19. Kabir A, Pourshams A, Khoshnia M, Malekzadeh F. Normal limit for serum alanine aminotransferase level and distribution of metabolic factors in old population of Kalaleh, Iran. Hepat Mon 2013, 13(10).

20. Bhatt SP, Nigam P, Misra A, Guleria R, Pandey RM, Pasha MQ. Genetic variation in the patatin-like phospholipase domain-containing protein-3 (PNPLA-3) gene in Asian Indians with nonalcoholic fatty liver disease. Metab Syndr Relat Disord 2013, 11(5):329-335.

21. Lin Y-C, Chang P-F, Hu F-C, Yang W-S, Chang M-H, Ni Y-H. A common variant in the PNPLA3 gene is a risk factor for non-alcoholic fatty liver disease in obese Taiwanese children. J Pediatr 2011, 158(5):740-744.

22. Hotta K, Yoneda M, Hyogo H, Ochi H, Mizusawa S, Ueno T, Chayama K, Nakajima A, Nakao K, Sekine A. Association of the rs738409 polymorphism in PNPLA3 with liver damage and the development of nonalcoholic fatty liver disease. BMC Med Genet 2010, 11(1):172.

23. Petta S, Miele L, Bugianesi E, Cammà C, Rosso C, Boccia S, Cabibi D, Di Marco V, Grimaudo S, Grieco A. Glucokinase regulatory protein gene polymorphism affects liver fibrosis in non-alcoholic fatty liver disease. PLoS One 2014, 9(2):e87523.

24. Shen J, Wong GH, Chan HY, Chan HY, Yeung DW, Chan RM, Chim AL, Chan AH, Choi PL, Woo J. PNPLA3 gene polymorphism accounts for fatty liver in community subjects without metabolic syndrome. Aliment Pharmacol Ther 2014, 39(5):532-539. 
Page 15/15 